\title{
Implementation of an Enhanced Recovery After Spine Surgery program at a large cancer center: a preliminary analysis
}

\author{
Roxana M. Grasu, MD, ${ }^{1}$ Juan P. Cata, MD,,5 Anh Q. Dang, MD, ${ }^{1}$ Claudio E. Tatsui, MD, ${ }^{2}$ \\ Laurence D. Rhines, MD, ${ }^{2}$ Katherine B. Hagan, MD, ${ }^{1}$ Shreyas Bhavsar, DO, ${ }^{1}$ \\ Sally R. Raty, MD, MHA, ${ }^{1}$ Radha Arunkumar, MD, ${ }^{1}$ Yury Potylchansky, MD, ${ }^{1}$ lan Lipski, MD, ${ }^{1}$ \\ Benjamin A. Arnold, MD, ${ }^{1}$ Thomas M. McHugh, MD, ${ }^{1}$ Justin E. Bird, MD, ${ }^{3}$ \\ Andrea Rodriguez-Restrepo, MD, ${ }^{6}$ Mike Hernandez, MS,${ }^{4}$ and Keyuri U. Popat, MD1
}

\begin{abstract}
Departments of ${ }^{1}$ Anesthesiology and Perioperative Medicine, ${ }^{2}$ Neurosurgery, ${ }^{3}$ Orthopedic Oncology, and ${ }^{4}$ Biostatistics, and ${ }^{5}$ Anesthesiology and Surgical Oncology Research Group, The University of Texas MD Anderson Cancer Center, Houston, Texas; and ${ }^{6}$ Department of Surgery, The University of Arizona, Tucson, Arizona
\end{abstract}

\begin{abstract}
OBJECTIVE Enhanced Recovery After Surgery (ERAS) programs follow a multimodal, multidisciplinary perioperative care approach that combines evidence-based perioperative strategies to accelerate the functional recovery process and improve surgical outcomes. Despite increasing evidence that supports the use of ERAS programs in gastrointestinal and pelvic surgery, data regarding the development of ERAS programs in spine surgery are scarce. To evaluate the impact of an Enhanced Recovery After Spine Surgery (ERSS) program in a US academic cancer center, the authors introduced such a program and hypothesized that ERSS would have a significant influence on meaningful clinical measures of postoperative recovery, such as pain management, postoperative length of stay (LOS), and complications.
\end{abstract}

METHODS A multimodal, multidisciplinary, continuously evolving team approach was used to develop an ERAS program for all patients undergoing spine surgery for metastatic tumors at The University of Texas MD Anderson Cancer Center from April 2015 through September 2016. This study describes the introduction of that ERSS program and compares 41 patients who participated in ERSS with a retrospective cohort of 56 patients who underwent surgery before implementation of the program. The primary objectives were to assess the effect of an ERSS program on immediate postoperative pain scores and in-hospital opioid consumption. The secondary objectives included assessing the effect of ERSS on postoperative in-hospital LOS, 30-day readmission rates, and 30-day postoperative complications.

RESULTS The ERSS group showed a trend toward better pain scores and decreased opioid consumption compared with the pre-ERSS group. There were no significant differences in LOS, 30-day readmission rate, or 30-day complication rate observed between the two groups.

CONCLUSIONS An ERSS program is feasible and potentially effective on perioperative pain control and opioid consumption, and can expedite recovery in oncological spine surgery patients. Larger-scale research on well-defined postoperative recovery outcomes is needed.

https://thejns.org/doi/abs/10.3171/2018.4.SPINE171317

KEYWORDS enhanced recovery; spine surgery; length of stay; postoperative pain; opioid consumption; outcomes

$\mathrm{T}$ 1 HE concept of Enhanced Recovery After Surgery (ERAS) has emerged as a method for substantially improving postoperative outcomes. ${ }^{16-19}$ The principle of ERAS is modulating and minimizing the surgical stress response and the associated increases in insulin re- sistance, hyperglycemia, and protein catabolism.,.5 ERAS integrates multimodal and multidisciplinary perioperative care protocols built on evidence-based perioperative care elements that individually have been shown to be beneficial in reducing the surgical stress response; when used

ABBREVIATIONS ASA = American Society of Anesthesiologists; AUC = area under the curve; DVT = deep vein thrombosis; ERAS = Enhanced Recovery After Surgery; ERSS = Enhanced Recovery After Spine Surgery; LITT = laser interstitial thermal therapy; LOS = length of stay; MEDD = morphine equivalent daily dose; MISS = minimally invasive spine surgery; NRS = numeric rating scale; POD = postoperative day; TIVA = total intravenous anesthesia.

SUBMITTED December 1, 2017. ACCEPTED April 2, 2018.

INCLUDE WHEN CITING Published online August 17, 2018; DOI: 10.3171/2018.4.SPINE171317. 
together in a single pathway, these individual elements can improve postoperative outcomes. ${ }^{27}$ The successful implementation of an ERAS program depends on coordination between all perioperative services involved in patient care across outpatient, in-hospital, and postdischarge phases. ${ }^{11,18,40}$ Additionally, constant evaluation of postoperative outcomes, including pain control, length of stay (LOS), complications, readmissions, and patient satisfaction, is essential for the program's ongoing data-driven quality improvement strategies. ${ }^{1,3,6,20,25,29,32,34,40}$

The volume and complexity of spine surgeries are rising worldwide. ${ }^{40}$ Despite improvements in surgical techniques, anesthesia, and perioperative care, major spine surgery is still associated with significant morbidity and postoperative pain, decreased postoperative functional recovery, and prolonged LOS. 3,7,15 There is increasing evidence of benefit from ERAS programs in gastrointestinal, pelvic, and orthopedic surgeries, such as hip and knee replacement, but there is little information on the development of an enhanced recovery pathway in spine surgery. ${ }^{28,40,41}$ Given the success of ERAS protocols in reducing morbidity and improving postoperative outcomes in other major surgeries, we hypothesized that an ERAS program tailored to patients undergoing spine surgery would have similar benefits. In our institution, we implemented a multimodal, multidisciplinary Enhanced Recovery After Spine Surgery (ERSS) program for patients undergoing spine surgery for metastatic tumors.

In this study, we reviewed postoperative outcomes before and after the implementation of ERSS. We hypothesized that the implementation of an ERSS program improved pain management, reduced postoperative LOS (a surrogate measure of postoperative inpatient recovery), and decreased the rate of postoperative complications.

\section{Methods}

\section{ERAS Program Components}

After reviewing the existing literature on successful ERAS programs and consulting with several national and international experts on ERAS, we formed a multidisciplinary ERSS committee comprising representatives in neuroanesthesia, neurosurgery, nursing, and other services, such as physical medicine and rehabilitation and nutrition. The committee developed a program built on an evidence-based approach to improve the perioperative care of patients undergoing spine surgery at The University of Texas MD Anderson Cancer Center. A standardized protocol was created to guide the perioperative management of spine surgery patients, and we prospectively collected perioperative data in a REDCap database..$^{10}$

The ERSS program was introduced over several months and considered fully operational by April 2015. The committee met weekly to discuss, revise, and monitor adherence to the protocol. Although the ERSS protocol was provided to and discussed with members of all the perioperative services, the provider ultimately decided whether to deviate from the protocol if deemed necessary or clinically indicated.

The ERSS protocol (Table 1) is a comprehensive plan covering the continuum of care from preoperative prepa- ration to intraoperative management to postoperative recovery. The preoperative protocol includes patient education, preconditioning exercise, optimization of comorbidities by the oncological primary care and specialty teams, and oral intake guidelines that allow patients to drink clear liquids up to 2 hours before the surgery. In the immediate preoperative period, patients receive oral analgesics consisting of acetaminophen $(1000 \mathrm{mg})$, extended-release tramadol $(300 \mathrm{mg})$, and either pregabalin $(75-150 \mathrm{mg})$ or gabapentin (100-300 mg) unless administration is contraindicated. Intraoperatively, the protocol recommends 1) total intravenous anesthesia (TIVA) with bispectral index monitoring for guidance on anesthesia depth; 2) infusions of lidocaine, ketamine, and dexmedetomidine; 3) regularly scheduled intravenous acetaminophen; 4) epidural analgesia or liposomal bupivacaine for surgical wound infiltration; 5) goal-directed fluid therapy and hemodynamic optimization; 6) restrictive blood transfusion and the use of tranexamic acid; 7) risk-based antiemetic prophylaxis; 8) deep vein thrombosis (DVT) prophylaxis; and 9) maintenance of normothermia. The intraoperative surgery element evolved toward a change in practice with the development, improvement, and preferential use of minimally invasive techniques when clinically appropriate. The postoperative component includes specific guidelines for minimization of opioid consumption, DVT prophylaxis, early oral intake starting on postoperative day (POD) 1, early ambulation, and physical therapy.

\section{Study Groups}

After IRB approval, we conducted a retrospective study of consecutive patients at our institution who underwent spine surgery for metastatic tumor resection before (preERSS group, or control group) and after (ERSS group) the implementation of the program in April 2015. We collected demographic and perioperative data from all patients in the study. We included patients $\geq 18$ years old who underwent spine surgery for metastatic spine tumors under general anesthesia whose medical records had complete postoperative pain data. The surgery type included open surgery such as decompression, stabilization, and/or vertebrectomy, and minimally invasive spine surgery (MISS) such as transcutaneous vertebroplasty and/or stabilization. We excluded patients with primary spine tumors, emergency surgery, laser interstitial thermal therapy (LITT) procedures, or incomplete pain score data. Patients in the pre-ERSS group typically received conventional balanced general anesthesia using a combination of volatile agents, intravenous anesthetics, and infusion of opioids according to clinical judgment. Both groups had similar preoperative optimization of comorbidities, management of complications, and hospital practice/discharge criteria.

\section{Outcome Measurement}

The primary outcomes were postoperative pain scores, on a numeric rating scale (NRS) of $0-10$, with 0 indicating no pain and 10 indicating the worst possible pain, and postoperative opioid consumption measured in oral morphine equivalent daily dose (MEDD). We assessed the primary outcomes for the postoperative periods of PODs 


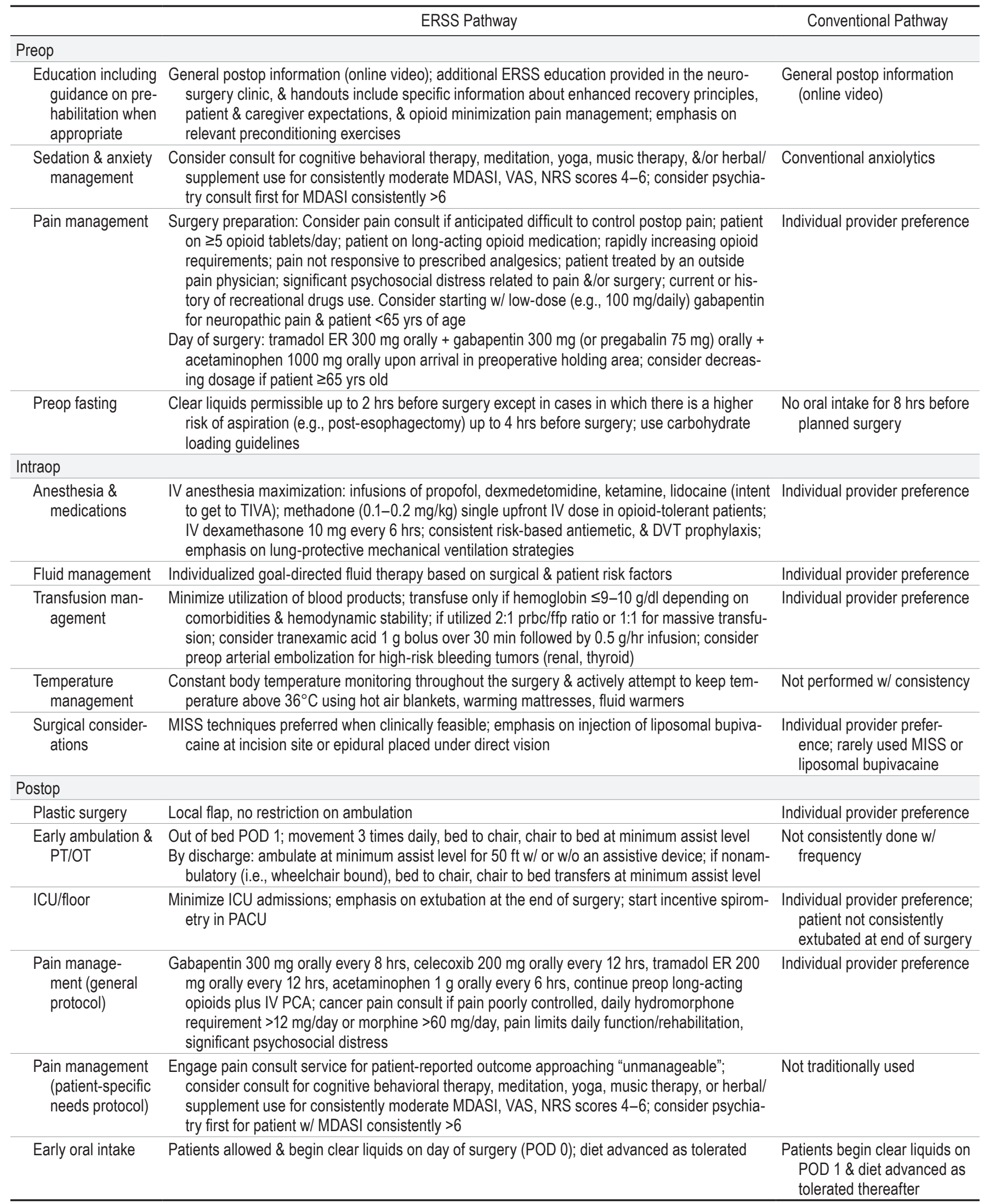

ffp = fresh frozen plasma; ICU = intensive care unit; IV = intravenous; MDASI = MD Anderson Symptom Inventory Scale, a multi-symptom patient-reported outcome measure for clinical and research use; PACU = postanesthesia care unit; PCA = patient-controlled analgesia; prbc = packed red blood cells; PT/OT = physical therapy/ occupational therapy; VAS = pain visual analog scale. 
0-3 and the discharge day. The secondary outcomes were postoperative hospital LOS, hospital readmission rate, and postoperative complications. Postoperative LOS was defined as the time from completion of surgery to hospital discharge. Readmission was defined as hospital readmission for any cause within 30 days of discharge. Postoperative complications were defined as any medical or surgical complications within 30 days of surgery.

\section{Statistical Analysis}

Descriptive statistics such as the mean (standard deviation) and median (range) summarized continuous variables. Frequencies and percentages summarized categorical variables. An independent samples t-test, or Wilcoxon rank-sum test if more appropriate, was used to compare continuous variables between ERSS and pre-ERSS patients. A chi-square test, or Fisher's exact test if more appropriate, was used to compare categorical variables between ERSS and pre-ERSS patients.

Intrapatient changes in pain scores were computed relative to POD 0 . The absolute changes were categorized as favorable (for positive changes), neutral (for zero difference), and unfavorable (for negative changes). Because varying numbers of pain assessments were elicited from patients, both maximum and average pain scores were captured to better quantify patients' pain experiences. For pain scores and total oral MEDD measured longitudinally over time, the area under the curve (AUC) was computed for patients using the trapezoidal rule. Patients with complete information from PODs $0-3$ and at discharge were used to compute the mean AUC, and mean AUCs were compared between ERSS and pre-ERSS patients. Spearman's rank correlation coefficient was used to assess the strength of association between the AUC for pain scores and the AUC for MEDD.

Because the distribution of LOS was highly skewed, a natural logarithmic transformation was applied to normalize the distribution. Linear regression was used followed by exponentiation of parameter estimates to acquire geometrical means associated with LOS. A p value $<0.05$ was considered statistically significant, and analyses were performed using Stata software (version 13, StataCorp).

\section{Results \\ Patient Population}

Of the 97 patients included, 41 patients were in the ERSS group and 56 patients were in the pre-ERSS group. Relevant patient demographic characteristics are shown in Table 2. Additional surgical characteristics are displayed in Table 3. No significant differences between groups (Table 2) were identified with regard to age, gender, American Society of Anesthesiologists (ASA) class, comorbidities, or smoking status. The mean $( \pm \mathrm{SD})$ BMI $\left(\mathrm{kg} / \mathrm{m}^{2}\right)$ was slightly lower in the ERSS group $\left(25.5 \pm 4.9 \mathrm{~kg} / \mathrm{m}^{2}\right)$ than in the pre-ERSS group $\left(28.5 \pm 5.7 \mathrm{~kg} / \mathrm{m}^{2}, \mathrm{p}=0.007\right)$. The ERSS group had a shorter surgical time $(263.9 \pm 128.0$ $\mathrm{min})$ than the pre-ERSS group $(315.8 \pm 152.1 \mathrm{~min}, \mathrm{p}=$ 0.080 ). The percentages of patients with preoperative pain were similar between groups $(90 \%$ in ERSS vs $93 \%$ in pre-ERSS, $p=0.644)$. The study surgery location and the location of the primary tumor were heterogeneous (Table 3 ). With respect to surgery location, thoracic (41\%), lumbar $(22 \%)$, and thoracolumbar (15\%) comprised the majority of locations observed. Kidney (21\%), lung (18\%), and breast $(10 \%)$ were the most common primary tumors. The ERSS group had more breast (12\% vs $8 \%$ ) and lung (22\% vs $16 \%$ ) tumors, while the pre-ERSS group had more kidney $(25 \%$ vs $15 \%, \mathrm{p}=0.698)$ primary tumors. The majority, and similar percentage, of patients in both groups had 1-stage surgery (96\%) and 3 or more spine levels operated (84\%). However, a significantly greater percentage of ERSS patients underwent MISS (27\% ERSS vs $2 \%$ pre-ERSS, $\mathrm{p}<0.001)$. The majority of open spine surgery categories observed included decompression only (19\%), decompression/stabilization (14\%), and decompression/ stabilization/vertebrectomy (41\%).

\section{Adherence of Providers to the Program's Preoperative and Intraoperative Multimodal Analgesia}

A higher percentage of patients in the ERSS group received preoperative nonopioid analgesics $(39.0 \%$ vs $10.7 \%, p=0.001$ ), including gabapentinoids and tramadol. With regard to anesthetic technique, the ERSS group used TIVA at a greater rate than the pre-ERSS group (22.0\% vs $7.1 \%, \mathrm{p}=0.050$ ). The ERSS group also showed increased intraoperative rates of lidocaine ( $42 \%$ vs $14 \%, p=0.003)$ and ketamine infusions (51\% vs $29 \%, \mathrm{p}=0.023)$, while the frequency of dexmedetomidine use was only slightly higher in the ERSS group than in the pre-ERSS group. The percentage of patients receiving epidural analgesia was not significantly different (ERSS $9.8 \%$ vs pre-ERSS $8.9 \%, \mathrm{p}=1.000)$. However, we observed more use of liposomal bupivacaine for infiltration of the surgical wound in the ERSS group ( $54 \%$ vs $30 \%, \mathrm{p}=0.021)$.

\section{Pain Scores and Opioid Consumption}

Table 4 summarizes the results for the primary and secondary outcome measures. By POD 1, there was some separation between the mean pain scores of the two groups, although this difference was not statistically significant. At POD $2(\mathrm{p}=0.065)$, POD $3(\mathrm{p}=0.027)$, and discharge, the ERSS group had lower average pain scores than the pre-ERSS group (Table 4, Fig. 1). A similar trend was identified for maximum pain scores, but statistical significance was not achieved (Table 4, Fig. 2). The proportion of patients experiencing superior pain control was higher for the ERSS group than the pre-ERSS group. Additionally, examination of the AUC using a complete case analysis indicated that the mean AUC for the average pain score experienced was significantly lower for ERSS patients ( $\mathrm{p}$ $=0.043$; Table 4 ).

The median total MEDD (PODs 0-3) was $372.2 \mathrm{mg}$ for the ERSS group and $521.5 \mathrm{mg}$ for the pre-ERSS group $(\mathrm{p}=0.507)$. Although the MEDD distribution was highly skewed and contained outliers, the ERSS patients received less total morphine. There was a significant Spearman's rank correlation ( $r h o=0.47, \mathrm{n}=78, \mathrm{p}<0.001$ ) between the AUC of average pain scores and total MEDD for PODs $0-3$. Additionally, a significant Spearman's rank correlation (rho $=0.42, \mathrm{n}=78, \mathrm{p}<0.001$ ) between the AUC of 
TABLE 2. Characteristics of study participants

\begin{tabular}{|c|c|c|c|c|}
\hline Characteristic & ERSS $(n=41)$ & Pre-ERSS $(n=56)$ & $p$ Value & Total $(n=97)$ \\
\hline Age, yrs & & & $0.591^{*}$ & \\
\hline Mean \pm SD & $58.8 \pm 11.3$ & $57.6 \pm 10.5$ & & $58.1 \pm 10.8$ \\
\hline Median (range) & $59(28-80)$ & $60(31-78)$ & & $59(28-80)$ \\
\hline Sex, no. $(\%)$ & & & $0.292 \dagger$ & \\
\hline Female & $19(46.3)$ & $20(35.7)$ & & $39(40.2)$ \\
\hline Male & $22(53.7)$ & $36(64.3)$ & & $58(59.8)$ \\
\hline $\mathrm{BMI}, \mathrm{kg} / \mathrm{m}^{2}$ & & & $0.007^{*}$ & \\
\hline Mean \pm SD & $25.5 \pm 4.9$ & $28.5 \pm 5.7$ & & $27.2 \pm 5.6$ \\
\hline Median (range) & $25.4(15.7-38.6)$ & $28.1(18.8-43.9)$ & & $27.4(15.7-43.9)$ \\
\hline Surgical time, $\min$ & & & $0.075 \S$ & \\
\hline Mean \pm SD & $263.9 \pm 128.0$ & $315.8 \pm 152.1$ & & $293.8 \pm 144.0$ \\
\hline Median (range) & $227(87-552)$ & $321.5(47-954)$ & & 275 (47-954) \\
\hline Preop pain, no. (\%) & & & $0.644 \dagger$ & \\
\hline No & $4(9.8)$ & $4(7.1)$ & & $8(8.2)$ \\
\hline Yes & $37(90.2)$ & $52(92.9)$ & & $89(91.8)$ \\
\hline ASA class, no. (\%) & & & $0.323 \ddagger$ & \\
\hline 2 & $1(2.4)$ & $1(1.8)$ & & $2(2.1)$ \\
\hline 3 & $38(92.7)$ & $55(98.2)$ & & $93(95.9)$ \\
\hline 4 & $2(4.9)$ & $0(0.0)$ & & $2(2.1)$ \\
\hline Chronic cardiovascular disease, no. (\%)ף & & & $0.572 \ddagger$ & \\
\hline No & $39(95.1)$ & $55(98.2)$ & & $94(96.9)$ \\
\hline Yes & $2(4.9)$ & $1(1.8)$ & & $3(3.1)$ \\
\hline Chronic pulmonary disease, no. (\%) & & & $0.393 \ddagger$ & \\
\hline No & $40(97.6)$ & $52(92.9)$ & & $92(94.8)$ \\
\hline Yes & $1(2.4)$ & $4(7.1)$ & & $5(5.2)$ \\
\hline Diabetes mellitus, no. (\%) & & & $0.395 \dagger$ & \\
\hline No & $35(85.4)$ & $44(78.6)$ & & $79(81.4)$ \\
\hline Yes & $6(14.6)$ & $12(21.4)$ & & $18(18.6)$ \\
\hline History of smoking, no. (\%) & & & $0.962 \dagger$ & \\
\hline Current/past* ${ }^{* *}$ & $6(14.6)$ & $8(14.3)$ & & $14(14.4)$ \\
\hline None & $35(85.4)$ & $48(85.7)$ & & $83(85.6)$ \\
\hline
\end{tabular}

* t-test.

† Chi-square test.

$\ddagger$ Fisher exact test.

$\S$ Wilcoxon rank-sum test.

T Chronic cardiovascular disease includes myocardial infarction, congestive heart failure, peripheral vascular disease, and cerebrovascular disease.

** Past smokers were defined as those who had quit smoking within 1 year.

maximum pain scores and total MEDD for PODs $0-3$ was noted. These analyses supported our finding that patients with higher pain trajectories characterized by the AUC also had higher total MEDDs (across PODs 0-3).

\section{Length of Stay, 30-Day Readmission Rate, and 30-Day Complication Rate}

Patients receiving ERSS had a geometrical mean LOS of $6.3 \pm 2.2$ days, while the pre-ERSS group had a slightly longer LOS of $6.8 \pm 1.9$ days $(p=0.590)$. The ratio of the pre-ERSS to ERSS geometrical means was approximately 1.08 (95\% confidence interval $0.81-1.43)$ and suggests that pre-ERSS patients stayed $8 \%$ longer in the hospital on average (Table 4). ERSS patients had a slightly higher 30-day hospital readmission rate than pre-ERSS patients (14.6\% vs $8.9 \%, \mathrm{p}=0.381$; Table 4 ).

The 30-day complication rate was $31.7 \%$ for ERSS patients and $17.9 \%$ for pre-ERSS patients $(\mathrm{p}=0.113$; Table 4). The most common 30-day complications were cardiovascular (17\%) and respiratory (9.7\%) for the ERSS group and infectious (7.1\%) and renal (5.4\%) for the pre-ERSS group.

\section{Discussion}

To our knowledge, the present study is the first to report the implementation of a multimodal, multidisciplinary, enhanced recovery program for spine surgery and to describe its influence on several meaningful recovery out- 
TABLE 3. Additional surgical characteristics

\begin{tabular}{|c|c|c|c|c|}
\hline Characteristic & $\begin{array}{l}\text { ERSS } \\
(n=41)\end{array}$ & $\begin{array}{l}\text { Control } \\
(n=56)\end{array}$ & $\begin{array}{c}p \\
\text { Value }\end{array}$ & $\begin{array}{c}\text { Total } \\
(n=97)\end{array}$ \\
\hline Surgery location & & & 0.322 & \\
\hline Cervical & $2(4.9)$ & $8(14.3)$ & & $10(10.3)$ \\
\hline Thoracic & $17(41.5)$ & $23(41.1)$ & & $40(41.2)$ \\
\hline Lumbar & $8(19.5)$ & $13(23.2)$ & & $21(21.6)$ \\
\hline Sacral & $1(2.4)$ & $1(1.8)$ & & $2(2.1)$ \\
\hline Cervical-thoracic & $1(2.4)$ & $4(7.1)$ & & $5(5.1)$ \\
\hline Thoracic-lumbar & $9(21.9)$ & $6(10.7)$ & & $15(15.5)$ \\
\hline Lumbar-sacral & $3(7.3)$ & $1(1.8)$ & & $4(4.1)$ \\
\hline Primary tumor location & & & 0.698 & \\
\hline Breast & $5(12.2)$ & $4(7.8)$ & & $9(9.8)$ \\
\hline Lung & $9(21.9)$ & $8(15.7)$ & & $17(18.5)$ \\
\hline Kidney & $6(14.6)$ & $13(25.5)$ & & $19(20.6)$ \\
\hline Colorectal & $1(2.4)$ & $3(5.9)$ & & $4(4.3)$ \\
\hline Melanoma & $3(7.3)$ & $2(3.9)$ & & $5(5.4)$ \\
\hline Lymphoma & $1(2.4)$ & $2(3.9)$ & & $3(3.3)$ \\
\hline Malignant bone tumor & $0(0)$ & $2(3.9)$ & & $2(2.2)$ \\
\hline Other* & $16(39.0)$ & $17(33.3)$ & & $33(35.9)$ \\
\hline Staged procedure & & & 0.636 & \\
\hline No & $40(97.6)$ & $53(94.6)$ & & $93(95.9)$ \\
\hline Yes & $1(2.4)$ & $3(5.4)$ & & $4(4.1)$ \\
\hline Spine levels operated & & & 0.412 & \\
\hline 1-2 levels & $5(12.2)$ & $11(19.6)$ & & $16(16.5)$ \\
\hline 3 or more & $36(87.8)$ & $45(80.4)$ & & $81(83.5$ \\
\hline Type of surgery & & & $<0.001$ & \\
\hline MISS & $11(26.8)$ & $1(1.8)$ & & $12(12.4)$ \\
\hline Open spine surgery & $30(73.2)$ & $55(98.2)$ & & $85(87.6)$ \\
\hline Decompression only & $6(14.6)$ & $12(21.4)$ & & $18(18.6)$ \\
\hline Stabilization only & $0(0.0)$ & $1(1.8)$ & & $1(1.0)$ \\
\hline $\begin{array}{l}\text { Decompression/ } \\
\text { stabilization }\end{array}$ & $4(9.8)$ & $10(17.9)$ & & $14(14.4)$ \\
\hline $\begin{array}{l}\text { Decompression/ } \\
\text { vertebrectomy }\end{array}$ & $2(4.9)$ & $3(5.4)$ & & $5(5.2)$ \\
\hline $\begin{array}{l}\text { Stabilization/verte- } \\
\text { brectomy }\end{array}$ & $3(7.3)$ & $4(7.1)$ & & $7(7.2)$ \\
\hline $\begin{array}{l}\text { Decompression/ } \\
\text { stabilization/ver- } \\
\text { tebrectomy }\end{array}$ & $15(36.6)$ & $25(44.6)$ & & $40(41.2)$ \\
\hline
\end{tabular}

All data given as number (percentage) unless otherwise indicated. The $p$ value was based on Fisher's exact test.

* Of the 33, 2 were unknown and 1 was not available.

comes. We found that the patients who underwent ERSS had a trend toward better pain scores and decreased opioid consumption compared with the pre-ERSS patients. We conclude that an ERSS program is feasible and potentially effective in improving postoperative outcomes such as pain control and opioid use in oncological spine surgery patients.

In a 2016 literature review, Wainwright et al. examined the evidence to support the application of individual ERAS components and concluded that an ERAS program could enable patients undergoing major spine surgery to recover more quickly with lower rates of morbidity and improved long-term outcomes. ${ }^{40}$ Some recent studies ${ }^{28,41}$ mentioned the development of an enhanced recovery approach for spine surgery; however, they each introduced and described only one ERAS intervention, such as multimodal pain management or an improvement in surgical technique. In a retrospective study, Mathiesen et al. reported that the implementation of a comprehensive, standardized multimodal pain protocol reduced postoperative opioid consumption and opioid-related adverse events and improved mobilization after multilevel spine surgery. ${ }^{28} \mathrm{In}$ a recent case series, Wang et al. reported the development and implementation of a MISS approach as part of an action plan for the development of an ERAS spinal fusion protocol. ${ }^{41}$

Our enhanced recovery program included a multitude of perioperative interventions (Table 1). Preoperatively, patient education, defined expectations, and active patient participation were essential to the success of the program. ${ }^{30}$ Additionally, we aimed to optimize preoperative pain management and reduce opioid consumption. ${ }^{4,9,14,21-23,31,35-37,39,42}$ Intraoperatively, anesthetic management included TIVA with propofol, dexmedetomidine, ketamine, and lidocaine infusions and scheduled intravenous acetaminophen for nonopioid analgesia. ${ }^{12,22,36,37}$ Other opioid-sparing pain management strategies included surgical wound infiltration with liposomal bupivacaine or epidural placement when indicated and scheduled high-dose intravenous dexamethasone to treat perioperative spinal cord edema. $9,21,23,35,41$

To maintain intraoperative normovolemia, ERSS uses goal-directed fluid therapy. ${ }^{39}$ Established guidelines for transfusion recommend only that providers avoid hemoglobin levels below $8-10 \mathrm{~g} / \mathrm{dl}$ depending on comorbidities and baseline preoperative hemoglobin. Patients with vascular tumors of the spine (e.g., renal or thyroid carcinoma metastases) undergo preoperative arterial embolization as previously described ${ }^{8,24}$ and intraoperative tranexamic acid administration. The importance of perioperative DVT prophylaxis, timely and appropriate antibiotic administration, glucose and temperature management, and lung-protective ventilator strategies was repeatedly emphasized to the team. The technological advances and improvement in surgical techniques, and particularly the development and implementation of MISS techniques, can significantly decrease perioperative morbidity and accelerate recovery. ${ }^{38,40,41}$ Therefore, MISS was recommended and preferred when clinically feasible.

For postoperative analgesia, patients received intravenous patient-controlled analgesia with a gradual transition to oral analgesics as needed. Scheduled high-dose steroids were continued, and oral acetaminophen, gabapentinoids, and tramadol were administered as soon as oral intake was tolerated. ${ }^{38}$ Early mobilization and diet advancement were implemented with strong support from physical medicine and rehabilitation services and from dieticians, respectively. ${ }^{26}$

Our results indicated that a trend toward better postoperative pain control correlated with a downward trend in total opioid consumption in the ERSS group. Reduced 
TABLE 4. Pain scores and morphine equivalent daily dose over time, LOS, 30-day hospital readmission rate, and 30-day postoperative complication rate

\begin{tabular}{|c|c|c|c|c|}
\hline Variable & ERSS $(n=41)$ & Pre-ERSS $(n=56)$ & $p$ Value & Total $(n=97)$ \\
\hline \multicolumn{5}{|l|}{ Max pain experienced } \\
\hline POD 0 & $n=41$ & $n=52$ & $0.832^{*}$ & $n=93$ \\
\hline Mean \pm SD & $5.4 \pm 3.3$ & $5.3 \pm 3.2$ & & $5.3 \pm 3.2$ \\
\hline Median (range) & $6(0-10)$ & $6(0-10)$ & & $6(0-10)$ \\
\hline POD 1 & $n=35$ & $n=54$ & $0.456^{*}$ & $n=89$ \\
\hline Mean \pm SD & $4.5 \pm 3.5$ & $5.0 \pm 3.3$ & & $4.8 \pm 3.4$ \\
\hline Median (range) & $4(0-10)$ & $6(0-10)$ & & $5(0-10)$ \\
\hline POD 2 & $n=38$ & $n=54$ & $0.216^{*}$ & $n=92$ \\
\hline Mean \pm SD & $4.0 \pm 3.0$ & $4.8 \pm 3.1$ & & $4.5 \pm 3.1$ \\
\hline Median (range) & $4(0-10)$ & $5.5(0-10)$ & & $5(0-10)$ \\
\hline POD 3 & $n=38$ & $n=53$ & $0.286^{*}$ & $\mathrm{n}=91$ \\
\hline Mean \pm SD & $4.0 \pm 2.8$ & $4.7 \pm 3.3$ & & $4.4 \pm 3.1$ \\
\hline Median (range) & $4(0-10)$ & $5(0-10)$ & & $4(0-10)$ \\
\hline Discharge day & $n=37$ & $n=54$ & $0.330^{*}$ & $\mathrm{n}=91$ \\
\hline Mean \pm SD & $2.8 \pm 2.9$ & $3.5 \pm 3.1$ & & $3.2 \pm 3.0$ \\
\hline Median (range) & $4(0-9)$ & $4(0-9)$ & & $4(0-9)$ \\
\hline Area under the curve & $n=34$ & $n=45$ & $0.183 \dagger$ & $n=79$ \\
\hline Mean \pm SD & $16.4 \pm 9.6$ & $19.4 \pm 9.6$ & & $18.1 \pm 9.7$ \\
\hline Median (range) & $15(2-36)$ & $20.5(0-35)$ & & $20(0-36)$ \\
\hline \multicolumn{5}{|l|}{ Avg pain experienced } \\
\hline POD 0 & $n=41$ & $n=52$ & $0.752^{*}$ & $n=93$ \\
\hline Mean \pm SD & $3.1 \pm 2.0$ & $3.0 \pm 2.3$ & & $3.0 \pm 2.1$ \\
\hline Median (range) & $3(0-6)$ & $3(0-8)$ & & $3(0-8)$ \\
\hline POD 1 & $n=35$ & $n=54$ & $0.232^{*}$ & $n=89$ \\
\hline Mean \pm SD & $2.5 \pm 2.2$ & $3.1 \pm 2.4$ & & $2.8 \pm 2.3$ \\
\hline Median (range) & $2(0-7)$ & $3(0-9)$ & & $3(0-9)$ \\
\hline POD 2 & $n=38$ & $n=54$ & $0.065^{*}$ & $n=92$ \\
\hline Mean \pm SD & $2.0 \pm 1.8$ & $2.8 \pm 2.2$ & & $2.4 \pm 2.1$ \\
\hline Median (range) & $2(0-7)$ & $3(0-8)$ & & $2(0-8)$ \\
\hline POD 3 & $n=38$ & $n=53$ & $0.027^{*}$ & $n=91$ \\
\hline Mean \pm SD & $1.6 \pm 1.7$ & $2.6 \pm 2.3$ & & $2.2 \pm 2.1$ \\
\hline Median (range) & $1.5(0-6)$ & $3(0-8)$ & & $2(0-8)$ \\
\hline Discharge day & $\mathrm{n}=37$ & $n=54$ & $0.311^{*}$ & $n=91$ \\
\hline Mean \pm SD & $1.5 \pm 1.9$ & $1.9 \pm 1.9$ & & $1.7 \pm 1.9$ \\
\hline Median (range) & $0(0-5)$ & $2(0-7)$ & & $1(0-7)$ \\
\hline AUC & $n=34$ & $n=45$ & $0.043 \dagger$ & $\mathrm{n}=79$ \\
\hline Mean \pm SD & $8.1 \pm 5.8$ & $11.2 \pm 6.8$ & & $9.9 \pm 6.5$ \\
\hline Median (range) & $7.2(0.5-21)$ & $11(0-27)$ & & $10(0-27)$ \\
\hline \multicolumn{5}{|l|}{ Total MEDD, mg } \\
\hline PODs 0-3 & $n=40$ & $n=55$ & $0.507 \dagger$ & $n=95$ \\
\hline Mean \pm SD & $678.9 \pm 954.2$ & $793.7 \pm 727.6$ & & $745.4 \pm 827.7$ \\
\hline Median (range) & $372.2(6-5426.5)$ & $521.5(31-2520)$ & & $409(6-5426.5)$ \\
\hline LOS, days & $n=41$ & $n=56$ & $0.590^{*}$ & $n=97$ \\
\hline Geometric mean \pm SD $\S$ & $6.3 \pm 2.2$ & $6.8 \pm 1.9$ & & $6.6 \pm 2.0$ \\
\hline Median (range) & $6(1-42)$ & $7(1-35)$ & & $6(1-42)$ \\
\hline 30-day readmissions, no. (\%) & & & $0.381 \ddagger$ & \\
\hline No & $35(85.4)$ & $51(91.1)$ & & $86(88.7)$ \\
\hline Yes & $6(14.6)$ & $5(8.9)$ & & $11(11.3)$ \\
\hline
\end{tabular}


» CONTINUED FROM PAGE 594

TABLE 4. Pain scores and morphine equivalent daily dose over time, LOS, 30-day hospital readmission rate, and 30-day postoperative complication rate

\begin{tabular}{llccc}
\hline \multicolumn{1}{c}{ Variable } & ERSS $(\mathrm{n}=41)$ & Pre-ERSS $(\mathrm{n}=56)$ & $\mathrm{p}$ Value & Total $(\mathrm{n}=97)$ \\
\hline 30-day complications, no. $(\%) \boldsymbol{T}$ & & & $0.113 \ddagger$ & \\
\hline No & $28(68.3)$ & $46(82.1)$ & & $74(76.3)$ \\
\hline Yes & $13(31.7)$ & $10(17.9)$ & $23(23.7)$ \\
\hline
\end{tabular}

Avg = average

${ }^{*}$ t-test.

$\dagger$ Wilcoxon rank-sum test.

$\ddagger$ Chi-square test.

§ Used owing to the skewness of the distribution of length of stay.

ๆ Complications included cardiovascular, respiratory, neurological, infectious, gastrointestinal, and renal complications.

pain can increase participation in physical therapy and rehabilitation with preservation of muscle strength and decreased incidence of DVT. ${ }^{13,16}$ Decreased opioid use should diminish the side effects of nausea, vomiting, constipation, postoperative ileus, sedation, and respiratory depression and should render the patient more functional and willing to participate in physical therapy. ${ }^{16-19,22,28,31,42}$

Postoperative hospital LOS, a secondary outcome, was chosen as a surrogate measure of postoperative inpatient recovery. In general, postprocedure recovery and early discharge are limited primarily by patient factors including age, comorbidities, severity of disease, complications, pain, opioid-related side effects, fatigue, and immobilization. LOS can also be influenced by medical facility factors, including practice style, availability of beds, and discharge policies. Thirty-day readmission rate was chosen as a measure to control for true readiness for discharge. The goal of an enhanced recovery program is to accelerate postoperative recovery by influencing all of the factors known to prolong LOS; therefore, LOS can serve as a surrogate measure for the effectiveness of such interventions. ${ }^{33}$

Although the pre-ERSS and ERSS groups in our study had similar demographic characteristics, comorbidities, category of surgery for metastatic spine tumor, and hospital practice/discharge criteria, and despite the ERSS group having significant MISS, shorter surgical time, and better pain management, we were not able to demonstrate a significant reduction in the mean LOS, readmissions, or complications in the ERSS group. This result may be explained by several reasons. First, our study included small groups of patients. In particular, the ERSS group included only 41 patients because this represented the first assessment of our recently introduced ERSS program and patients with LITT procedures or incomplete pain score data were excluded. Second, metastatic cancer patients with advanced disease or terminal illness can have a prolonged LOS after surgery to continue cancer treatment unrelated to the surgical procedure they were admitted for; therefore it was difficult to capture the patient's true readiness for discharge after the procedure. Third, we were not able to study and control for the severity of the oncological disease. Fourth, although the minimally invasive surgical techniques, consistently applied after the development of the ERSS program, were captured in the study, we excluded the LITT cases because the minimal invasiveness of this procedure could significantly influence the outcomes in our smallsized study. The potentially high impact of various types of MISS on the speed of recovery for these patients necessitates further research and larger-scale studies.

Success and sustainability of ERAS programs are based on level of compliance with an established protocol. Although our study indicated an increase in pre- and intraoperative multimodal analgesia in the ERSS group, the compliance rate for preoperative nonopioid analgesia (39\%) and other intraoperative analgesia interventions (epidural placement) was quite low. Our preliminary data, which clearly showed differences in individual provider preference for analgesia interventions, were used to increase provider's awareness, improve communication, and reinforce group compliance with the protocol. In our institution, we have several ERAS programs in various stages of development for oncological surgery, including colorectal, pancreatic, thoracic, urological, brain, and spine. Strategies such as increased collaboration among all perioperative services, institutional leadership involvement, growing institutional electronic ERAS databases and research, periodic program assessments and newsletters, ERAS conferences, and grand rounds contribute to increase providers' awareness, participation, and compliance with the protocols. Our study served as a stepping stone for further improvement of the ERSS protocol and group compliance, identifying relevant patient and surgical variables, updating our ERSS database, and defining meaningful postoperative outcomes for future larger-scale studies.

\section{Limitations of the Study}

Due to our study's retrospective design, the results should be interpreted with caution, especially when considering the use of historical data in characterizing the pre-ERSS group. Because the ERSS program was evidence-based and directed toward constant quality improvement, we had to select our pre-ERSS group from patients treated before the implementation of the full ERSS protocol. Despite this selection criterion, some patients in the pre-ERSS group could have already received certain elements of the ERSS protocol before formalization and implementation. However, in both the pre- and postintervention groups, the metastatic type of spine surgery and 


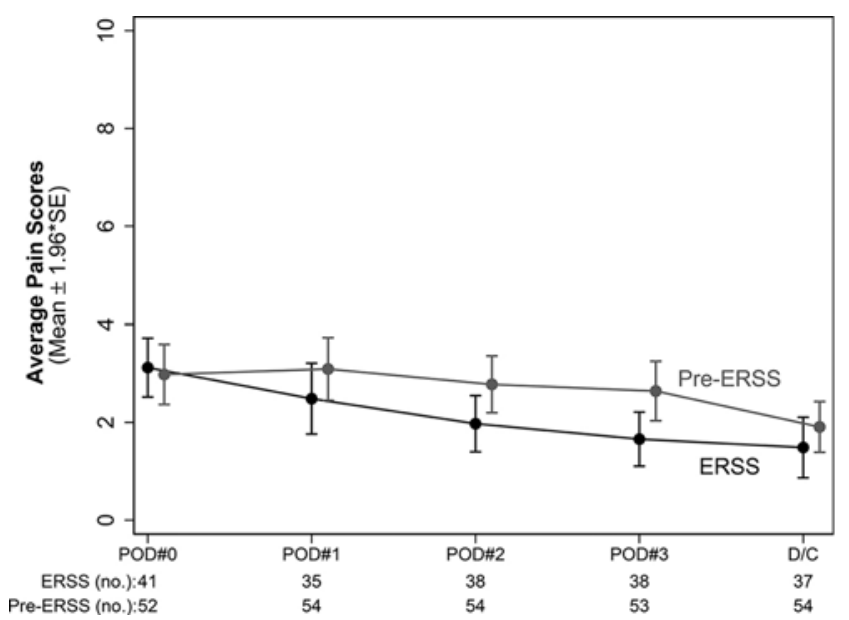

FIG. 1. Profile plots of the average pain scores across postoperative time by study cohorts (ERSS vs pre-ERSS). The pain was measured on an NRS (0-10) and postoperative time was defined across points in time, starting with day of surgery (POD 0 ), PODs $1-3$, and day of discharge $(D / C)$. In general, the trajectories of average pain scores display a decline on PODs 1-3 and by day of discharge. Although the findings were significant only on POD $3(p=0.027)$, the trajectories show more separation from POD 1 to POD 3 in favor of the ERSS group and the pain trajectory remains lower for ERSS patients from POD 1 to D/C. I bars indicate 1.96 times the standard error (SE) of the mean. Values below the graph indicate the number of patients remaining in the study at each time point.

discharge criteria in the department did not change, and the inconsistency of pain data was mitigated by our selection criteria and our ability to capture opioid consumption.

Our original query of the institutional database yielded 300 consecutive patients who had spine surgery for metastatic tumors. However, only a small number of patients within each study cohort met the inclusion criteria and provided analyzable data. Furthermore, the study describes only the first assessment of a recently implemented ERSS program. Although the number of ERSS patients was limited, patients on average were similar demographically between study cohorts. With a BMI lower than $30 \mathrm{~kg} / \mathrm{m}^{2}$ in both groups, the difference, although statistically significant, was considered too small to be clinically relevant. However, general surgical characteristics, such as type of primary tumor and magnitude of surgery, were heterogeneous. For example, more renal cancer spinal metastases in the pre-ERSS group can potentially increase the risk for bleeding and complications, and prolong LOS. Additionally, the shift in our surgical practice and emphasis on minimally invasive techniques when clinically indicated resulted in a significant percentage of ERSS patients having MISS, which can explain the decrease in magnitude of surgery and surgical time in ERSS versus the pre-ERSS group. Unfortunately, the small sample size in this study restricted our ability to conduct propensity score matching and rigorous analyses to measure the effects of ERSS while adjusting for covariates of interest.

We did not track other outcomes of enhanced recovery, such as early mobilization, recovery of bowel and urinary bladder function, or patient satisfaction, because information about specific mobilization steps, time to first meal,

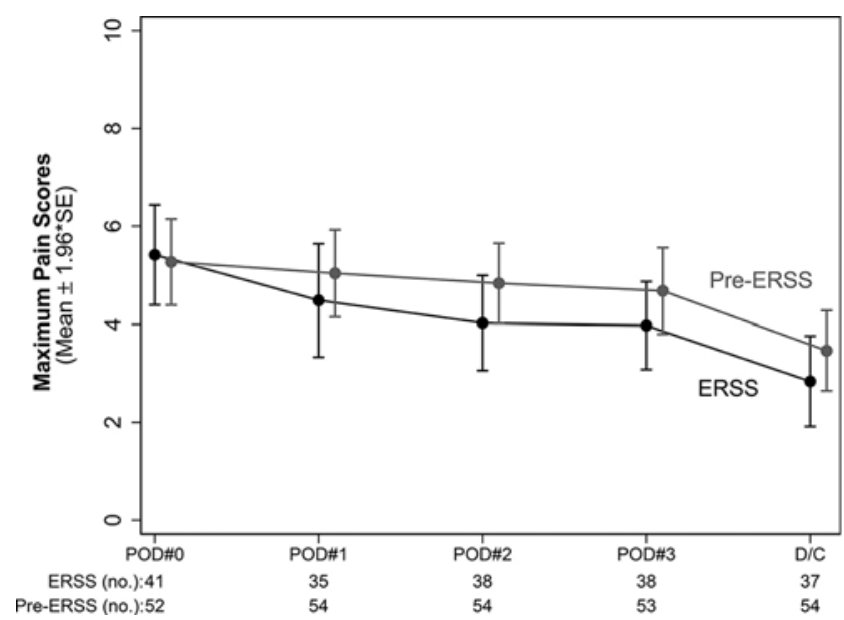

FIG. 2. Profile plots of the maximum pain scores across postoperative time by study cohorts (ERSS vs pre-ERSS). The pain was measured on an NRS (0-10) and postoperative time was defined across points in time, starting with day of surgery (POD 0 ), PODs $1-3$, and day of discharge $(D / C)$. In general, the trajectories of maximum pain scores show slow decline over PODs 1-3, and more decline by day of discharge. Although the findings were not significant and the trajectories show little separation in ERSS vs pre-ERSS groups, the pain trajectory remains slightly lower for ERSS patients from POD 0 to day of discharge. I bars indicate 1.96 times the standard error (SE) of the mean. Values below the graph indicate the number of patients remaining in the study at each time point.

duration of urinary catheterization, and patient experience was not consistently available. Our future studies will aim to assess not only patient satisfaction and patient-reported outcomes, but also the financial impact of our accelerated recovery program compared with traditional care. We believe that the ever-changing healthcare landscape and pressure to move toward high-quality value-based care will drive the impetus for advancing programs such as ERAS, which are aimed at accelerating patients' recovery while preserving or increasing quality of care.

Although we have shown a trend toward improvement in postoperative outcomes such as pain scores and opioid use, we were unable to demonstrate a significant reduction in LOS or postoperative complications or to measure the specific impact of individual components of the program, such as the MISS. The constant quality improvement and multiinterventional nature of an ERAS program constitutes one of the most challenging aspects of research on postoperative outcomes. Larger-scale studies on individual components and well-defined postoperative recovery outcomes are needed to assess the benefits of an ERAS program for spine surgery.

\section{Conclusions}

The implementation of a multidisciplinary, evidencebased ERAS program can be associated with improved analgesia and decreased opioid consumption in the perioperative care of patients undergoing spine surgery for metastatic tumors. Although retrospective, this study represents the first evaluation of a newly developed enhanced recovery program in oncological spine surgery and serves 
as a roadmap for further initiatives. More research aimed at capturing meaningful, reportable, patient-specific, and patient-reported outcomes, and larger-scale studies are needed to strengthen and validate our findings and demonstrate the benefits of the program. Our experience suggests that an enhanced recovery standardized protocol can be implemented system-wide in spine surgery. The adoption of such programs in spine surgery is feasible, can be beneficial, and should be encouraged.

\section{Acknowledgments}

The University of Texas MD Anderson Cancer Center is supported in part by the NIH through Cancer Center support grant no. P30CA016672.

\section{References}

1. Boltz MM, Hollenbeak CS, Ortenzi G, Dillon PW: Synergistic implications of multiple postoperative outcomes. Am J Med Qual 27:383-390, 2012

2. Carli F: Physiologic considerations of Enhanced Recovery After Surgery (ERAS) programs: implications of the stress response. Can J Anaesth 62:110-119, 2015

3. Deyo RA, Mirza SK, Martin BI, Kreuter W, Goodman DC, Jarvik JG: Trends, major medical complications, and charges associated with surgery for lumbar spinal stenosis in older adults. JAMA 303:1259-1265, 2010

4. Dunn LK, Durieux ME, Nemergut EC: Non-opioid analgesics: Novel approaches to perioperative analgesia for major spine surgery. Best Pract Res Clin Anaesthesiol 30:79-89, 2016

5. Fearon KCH: Overview: key elements and the impact of enhanced recovery care, in Francis N, Kennedy RH, Ljungsqvist O, et al (eds): Manual of Fast Track Recovery for Colorectal Surgery. London: Springer, 2012, pp 1-13

6. Ghaferi AA, Birkmeyer JD, Dimick JB: Variation in hospital mortality associated with inpatient surgery. N Engl J Med 361:1368-1375, 2009

7. Gillissen F, Hoff C, Maessen JM, Winkens B, Teeuwen JH, von Meyenfeldt MF, et al: Structured synchronous implementation of an enhanced recovery program in elective colonic surgery in 33 hospitals in The Netherlands. World J Surg 37:1082-1093, 2013

8. Gottfried ON, Schloesser PE, Schmidt MH, Stevens EA: Embolization of metastatic spinal tumors. Neurosurg Clin $\mathbf{N}$ Am 15:391-399, 2004

9. Grieff AN, Ghobrial GM, Jallo J: Use of liposomal bupivacaine in the postoperative management of posterior spinal decompression. J Neurosurg Spine 25:88-93, 2016

10. Harris PA, Taylor R, Thielke R, Payne J, Gonzalez N, Conde JG: Research electronic data capture (REDCap) - a metadata-driven methodology and workflow process for providing translational research informatics support. J Biomed Inform 42:377-381, 2009

11. Husted H: Fast-track hip and knee arthroplasty: clinical and organizational aspects. Acta Orthop Suppl 83:1-39, 2012

12. Hwang W, Lee J, Park J, Joo J: Dexmedetomidine versus remifentanil in postoperative pain control after spinal surgery: a randomized controlled study. BMC Anesthesiol 15:21, 2015

13. Jørgensen CC, Jacobsen MK, Soeballe K, Hansen TB, Husted $\mathrm{H}$, Kjærsgaard-Andersen $\mathrm{P}$, et al: Thromboprophylaxis only during hospitalisation in fast-track hip and knee arthroplasty, a prospective cohort study. BMJ Open 3:e003965, 2013

14. Joshi GP, Bonnet F, Kehlet H: Evidence-based postoperative pain management after laparoscopic colorectal surgery. Colorectal Dis 15:146-155, 2013
15. Kanaan SF, Waitman LR, Yeh HW, Arnold PM, Burton DC, Sharma NK: Structural equation model analysis of the length-of-hospital stay after lumbar spine surgery. Spine J 15:612-621, 2015

16. Kehlet H: Multimodal approach to control postoperative pathophysiology and rehabilitation. Br J Anaesth 78:606617, 1997

17. Kehlet H: [Painless and risk-free surgery-a vision of the future?] Ugeskr Laeger 156:3468-3469, 1994 (Danish)

18. Kehlet H, Dahl JB: Anaesthesia, surgery, and challenges in postoperative recovery. Lancet 362:1921-1928, 2003

19. Kehlet H, Wilmore DW: Evidence-based surgical care and the evolution of fast-track surgery. Ann Surg 248:189-198, 2008

20. Khuri SF, Henderson WG, Daley J, Jonasson O, Jones RS, Campbell DA Jr, et al: The patient safety in surgery study: background, study design, and patient populations. J Am Coll Surg 204:1089-1102, 2007

21. Kim J, Burke SM, Kryzanski JT, Roberts RJ, Roguski M, Qu $\mathrm{E}$, et al: The role of liposomal bupivacaine in reduction of post-operative pain after transforaminal lumbar interbody fusion (TLIF): a clinical study. World Neurosurg 91:460-467, 2016

22. Kim KT, Cho DC, Sung JK, Kim YB, Kang H, Song KS, et al: Intraoperative systemic infusion of lidocaine reduces postoperative pain after lumbar surgery: a double-blinded, randomized, placebo-controlled clinical trial. Spine J 14:1559-1566, 2014

23. Kjærgaard M, Møiniche S, Olsen KS: Wound infiltration with local anesthetics for post-operative pain relief in lumbar spine surgery: a systematic review. Acta Anaesthesiol Scand 56:282-290, 2012

24. Ladner TR, He L, Lakomkin N, Davis BJ, Cheng JS, Devin CJ, et al: Minimizing bleeding complications in spinal tumor surgery with preoperative Onyx embolization via dual-lumen balloon catheter. J Neurointerv Surg 8:210-215, 2016

25. Lawson EH, Hall BL, Louie R, Ettner SL, Zingmond DS, Han L, et al: Association between occurrence of a postoperative complication and readmission: implications for quality improvement and cost savings. Ann Surg 258:10-18, 2013

26. Lee TG, Kang SB, Kim DW, Hong S, Heo SC, Park KJ: Comparison of early mobilization and diet rehabilitation program with conventional care after laparoscopic colon surgery: a prospective randomized controlled trial. Dis Colon Rectum 54:21-28, 2011

27. Ljungqvist O: ERAS - enhanced recovery after surgery: moving evidence-based perioperative care to practice. JPEN J Parenter Enteral Nutr 38:559-566, 2014

28. Mathiesen O, Dahl B, Thomsen BA, Kitter B, Sonne N, Dahl JB, et al: A comprehensive multimodal pain treatment reduces opioid consumption after multilevel spine surgery. Eur Spine J 22:2089-2096, 2013

29. Polk HC Jr, Birkmeyer J, Hunt DR, Jones RS, Whittemore AD, Barraclough B: Quality and safety in surgical care. Ann Surg 243:439-448, 2006

30. Rawlinson A, Kang P, Evans J, Khanna A: A systematic review of enhanced recovery protocols in colorectal surgery. Ann R Coll Surg Engl 93:583-588, 2011

31. Rivkin A, Rivkin MA: Perioperative nonopioid agents for pain control in spinal surgery. Am J Health Syst Pharm 71:1845-1857, 2014

32. Rowell KS, Turrentine FE, Hutter MM, Khuri SF, Henderson WG: Use of national surgical quality improvement program data as a catalyst for quality improvement. J Am Coll Surg 204:1293-1300, 2007

33. Sarin A, Litonius ES, Naidu R, Yost CS, Varma MG, Chen LL: Successful implementation of an Enhanced Recovery After Surgery program shortens length of stay and improves postoperative pain, and bowel and bladder function after colorectal surgery. BMC Anesthesiol 16:55, 2016 
34. Schifftner TL, Grunwald GK, Henderson WG, Main D, Khuri SF: Relationship of processes and structures of care in general surgery to postoperative outcomes: a hierarchical analysis. J Am Coll Surg 204:1166-1177, 2007

35. Servicl-Kuchler D, Maldini B, Borgeat A, Bilić N, Kosak R, Mavcic B, et al: The influence of postoperative epidural analgesia on postoperative pain and stress response after major spine surgery - a randomized controlled double blind study. Acta Clin Croat 53:176-183, 2014

36. Smith AN, Hoefling VC: A retrospective analysis of intravenous acetaminophen use in spinal surgery patients. Pharm Pract (Granada) 12:417, 2014

37. Song JW, Shim JK, Song Y, Yang SY, Park SJ, Kwak YL: Effect of ketamine as an adjunct to intravenous patientcontrolled analgesia, in patients at high risk of postoperative nausea and vomiting undergoing lumbar spinal surgery. $\mathbf{B r} \mathbf{J}$ Anaesth 111:630-635, 2013

38. Tatsui CE, Stafford RJ, Li J, Sellin JN, Amini B, Rao G, et al: Utilization of laser interstitial thermotherapy guided by real-time thermal MRI as an alternative to separation surgery in the management of spinal metastasis. J Neurosurg Spine 23:400-411, 2015

39. Thiele RH, Raghunathan K, Brudney CS, Lobo DN, Martin D, Senagore A, et al: American Society for Enhanced Recovery (ASER) and Perioperative Quality Initiative (POQI) joint consensus statement on perioperative fluid management within an enhanced recovery pathway for colorectal surgery. Perioper Med (Lond) 5:24, 2016

40. Wainwright TW, Immins T, Middleton RG: Enhanced recovery after surgery (ERAS) and its applicability for major spine surgery. Best Pract Res Clin Anaesthesiol 30:91-102, 2016

41. Wang MY, Chang PY, Grossman J: Development of an enhanced recovery after surgery (ERAS) approach for lumbar spinal fusion. J Neurosurg Spine 26:411-418, 2017
42. Yu L, Ran B, Li M, Shi Z: Gabapentin and pregabalin in the management of postoperative pain after lumbar spinal surgery: a systematic review and meta-analysis. Spine (Phila Pa 1976) 38:1947-1952, 2013

\section{Disclosures}

Dr. Rhines reports being a consultant to Stryker and Medtronic.

\section{Author Contributions}

Conception and design: Grasu, Cata, Dang, Popat. Acquisition of data: Grasu, Cata, Dang, Hagan, Bhavsar, Raty, Arunkumar, Potylchansky, Lipski, Arnold, McHugh, Rodriguez-Restrepo, Popat. Analysis and interpretation of data: Grasu, Cata, Dang, Tatsui, Rhines, Bird, Hernandez, Popat. Drafting the article: Grasu. Critically revising the article: Grasu, Cata, Dang, Tatsui, Rhines, Hagan, Bhavsar, Raty, Arunkumar, Potylchansky, Lipski, Arnold, McHugh, Bird, Popat. Reviewed submitted version of manuscript: Grasu, Cata, Dang, Tatsui, Rhines, Hagan, Bhavsar, Raty, Arunkumar, Potylchansky, Lipski, Arnold, McHugh, Bird, RodriguezRestrepo, Popat. Approved the final version of the manuscript on behalf of all authors: Grasu. Statistical analysis: Hernandez. Administrative/technical/material support: Rodriguez-Restrepo, Hernandez. Study supervision: Grasu, Popat.

\section{Correspondence}

Roxana M. Grasu: The University of Texas MD Anderson Cancer Center, Houston, TX.rgrasu@mdanderson.org. 